\title{
Research on BP-ANN Model of Semi-rigid Connection
}

\author{
Jian Liu ${ }^{1}$, Xiangyun Huang ${ }^{1}$, Guangen Zhou ${ }^{2}$, Jiping $\mathrm{Hao}^{3}$, Da Ren ${ }^{1}$ and Yue Gao ${ }^{1}$ \\ 1. School of Civil Engineering, Guangzhou University, Guangzhou, China \\ 2. Postdoctoral Research Station, Zhejiang Southeast Lattice Frame Group, Zhejiang, China \\ 3. College of Civil Engineering, Xi'an University of Architecture and Technology, Xi'an, China
}

\begin{abstract}
The beam-to-column semirigid connection in a steel frame structure is represented by a zero-length rotational spring at the end of the beam element. The beam-to-column semirigid connection behavior is represented by its moment-rotation relationship. Several traditional mathematical models have been proposed to fit the moment-rotation curves from the experimental database,but they may be more reliable within certain ranges. In this paper, the intellectualized analytical model is proposed in the semirigid connections for top and seat angles with double web angles using the feed-forward back-propagation artificial neural network (BP-ANN) technique. the intellectualized analytical model from experimental results based on BP-ANN is more reliable and it is a better choice to the moment-rotation curves for beam-to-column semirigid connection. The results are found to provide effectiveness to the experimental response that is satisfactory for use in steel structural engineering design.
\end{abstract}

Key words: beam-to-column joint, semirigid connection, intellectualized analytical model, artificial neural network.

\section{Introduction}

Most steel structural design engineers assume the behavior of steel beam-column connections either as perfectly pinned or as completely fixed elements. This simplification results in an inaccurate prediction of frame behavior. Beams and columns in steel structure are generally connected with welding or high-strength bolting.

There are commonly three manners of joints connections between beams and columns for steel structures engineering design to be chosen, i.e., welding rigid connection between the beam and the column; high-strength bolting connection between the beam and the column;welding and bolting mixed rigid connection between the beam and the column. Configuration of pinned connections is relatively simple, but their stiffness is low, which is disadvantageous to aseismic design. For the welding connection between the beam and the column, and

Corresponding author: Jian Liu, $\mathrm{PhD}$, research fields: stability analysis and advanced analysis of semi-rigid connections steel structures and so on. E-mail: liuj5000@163.com. welding and bolting mixed connection between the beam and the column, rigid connection assumption, as shown in Fig. 1, with no relative rotation between the beam and the column can be adopted in steel structural analysis because of the sufficient rotation stiffness of the connection.

Semi-rigid beam-column connections, however, are the compromise of rigid and pinned connections, Bolted connections are popular in steel structures due to economy and simplicity in fabrication. The conventional design procedure for structural calculation assumes that the joints are pinned or completely rigid. However, in reality most joints in the steel structures are semi-rigid. The joint behavior between beams and columns makes an important contribution to the behavior of the whole structure, and the performance of the joints between beams and columns has been one of the critical factors in many structural designs.

Beam-to-column connections commonly used may be categorized as Ref. [1], single web angles,double web angles, header plates, top and seat angles, top and seat angles with double web angles, end-plates, and T-stubs. Among the various possible deformation 
modes for semi-rigid connections, the most important mode is rotational deformation caused by a bending moment. The basic description of semi-rigid behavior between the beam and the column is its moment-rotation relationship, which may be represented as the joint semi-rigid rigidity. Applied to the in-plane bending moments at beam ends, the primary deformation of steel beam-to-column connections is dominant by relative rotations. Typical moment-rotation relationship results from full-scale tests show this relationship to be nonlinear.

The neural network theory, which provides a solid basis for the construction of the model estimator, is considered highly suitable for the study of complex problems in mechanics and engineering. Some recent representative applications are reported in the area of structural analysis and design parameter identification problems, material modelling, structural analysis and optimization problems.

The semirigid connection between beams and columns is commonly modeled as zero-length rotational springs at the ends of a beam. The semirigid connection behavior between beams and columns is represented by its moment-rotation relationship. Several traditional mathematical models of the beam-to-column semi-rigid connections have been

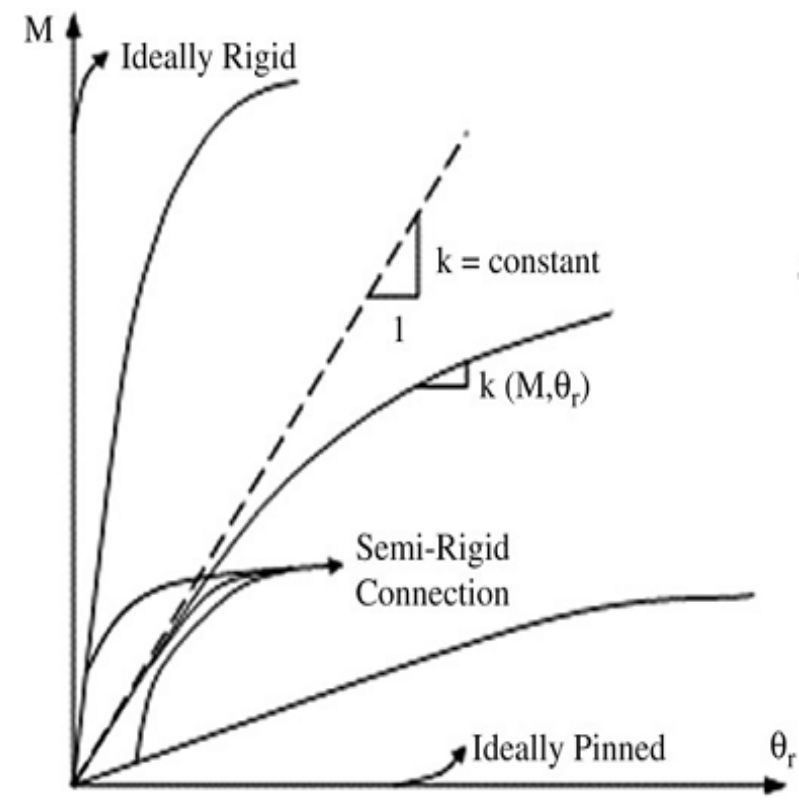

Fig. 1 Classification for connection. proposed to fit the moment-rotation curves from the experimental database,but they may be more reliable within certain ranges.In this paper, the intellectualized analytical model is proposed in semirigid connections for the top and seat angles with double web angles using the feed-forward BP-ANN technique.

\section{BP Artificial Neural Networks}

The system for Artificial neural networks has three layers of neurons: input layer, a hidden layer and an output layer. The neurons or units of the network are connected by the weights. The input layer consists of all the input factors, information from the input layer is then processed through one hidden layer, and following output vector is computed in the output layer. The scheme of ANN used in the paper is shown in Fig. 2.

Back propagation, which is one of the most famous training algorithms for multi-layer perceptions, is a gradient descent technique to minimize the error for particular training pattern. BP training algorithm was preferred due to its simplicity and reliability [2]. Each input unit of the input layer receives input signal $X_{i}$ and broadcasts this signal to all units in the hidden layer. Each hidden unit $Y_{j}$ sums its weighted input signal and applies its activation function to compute output signal.

$$
Y_{j}=f_{j}\left(\sum_{i=1}^{n} W_{i j} X_{i}+\theta_{j}\right)
$$

where $W_{i j}$ is the weight of the connection from the ith input unit to the $j$ th hidden unit, $\theta_{j}$ is the weight of bias connection for jth hidden unit. The output signal of the hidden unit $Y_{j}$ is sent to all units in the output layer. Each output unit $O k$ sums its weighted input signal and

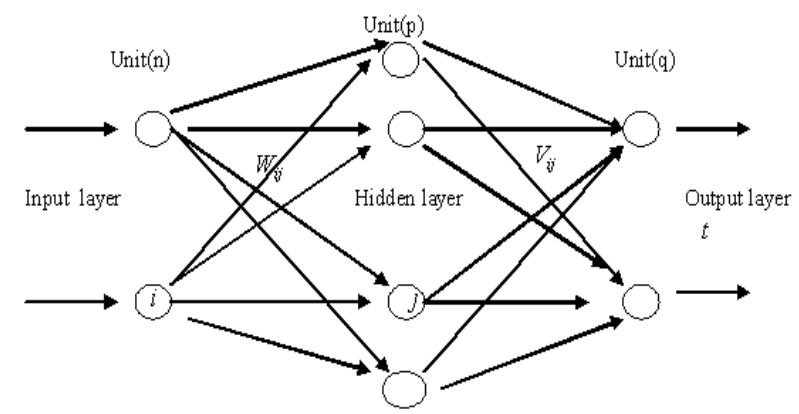

Fig. 2 Three-layer neural networks. 
applies its activation function to compute its output signal.

$$
O_{k}=f_{j}\left(\sum_{j=1}^{q} V_{j k} Y_{j}+\theta_{k}\right)
$$

where $\boldsymbol{V}_{j k}$ is the weight of the connection from the $j t h$ hidden unit to the $k$ th output unit. The parameter of bias $(\theta)$ in Eqs. (1) and (2), also called the threshold value, is permanently set to 1 in hidden layer as well as output layer so that corresponding weight shifts the activation function along the $X$ axis. The activation function used in this study is a logistic sigmoid function defined as.

$$
\mathrm{f}_{j}(\mathrm{x})=\frac{1}{1+\mathrm{e}^{-\mathrm{x}}}
$$

The BP-ANN training algorithm is an iterative gradient descent algorithm, designed to minimize the sum of square error $(E)$ which is averaged over all patterns and is calculated as follows

$$
E=\frac{1}{2} \sum_{p=1}^{p} \sum_{k=1}^{q}\left(d T_{p k}-O_{p k}\right)^{2}
$$

where $d T_{p k}$ is the desired or actual output, $O p k$ is the predicted output for the pattern. During training, an ANN is the presented with the data for thousands of times, which is referred to as cycles.After each cycle the error between the BP-ANN output and desired values are propagated backward to adjust the weight in a manner mathematically guarantied to converge. Adjustment of the weights $\triangle W_{i j}$ can be calculated as

$$
\Delta W_{i j}=-\eta \partial E / \partial W_{i j}+\alpha \Delta W_{i j}(n-1)
$$

where $\eta$ is the learning rate, $\alpha$ is the momentum coefficient and $n$ is the current step.

\section{The Modelling of Semirigid Connections}

As the behaviour of semi-rigid connections between beams and columns evidently affects the stiffness and load-carrying capacity of steel structures, clarifying the moment and rotation relationship of semirigid connections between beams and columns is a key point to second-order nonlinear analysis and design of steel structures involving such connections.

Connections act as a medium through which forces are transferred from one structural member to another.
The major force transferred through the connection is the moment, resulting in a rotational deformation. A connection under an applied moment rotates through angle, which is the angle between beam and column from their original position. The moment-rotation relationship shows the relative moment-rotation behavior of a variety of commonly used semirigid connections.

The moment-rotation relationship has been the subject of several studies. Full scale experiments are generally necessary to describe the actual behavior of these connections. Extensive experimental tests have been conducted on the commonly used connections, and a large collection of test data reported (Kishi and Chen 1986), based on previous investigations,Using these abundant data base,researchers have developed several connection mathematical models.Several models have been proposed to fit the moment-rotation curves from the experimental data. These models vary widely in their complexity, ranging from the simplest linear model to polynomial model, Cubic B-spline model, exponential model, and power model, the three parameter poewer model proposed by Kishi and Chen (1990). Several traditional mathematical models [1] have been proposed to fit the moment-rotation curves from the experimental data, but they may be more reliable within certain ranges.

\section{The BP-ANN Model of Connections}

BP-ANN is an artificial intelligence application implemented by engineers to carry out design tasks. It has been applied to problems of: predicting function approximation; structural analysis, design, and structural damage assessment, and so on.The BP-ANN are basically computational models, which simulate the function biological networks, composed of neurons. In the literature most papers on the use of artificial neural networks apply a multilayered, feed forward, fully connected network of perceptions. Among the reasons for using this kind of BP-ANN is the simplicity of its theory, ease of programming and good results. 
This paper utilizes experimental database for semi-rigid connections of between beams and columns to develop the Intellectualized prediction model based on the feed-forward back-propagation artificial neural network. The BP-ANN prediction model is designed to predict the moment and rotation curves semi-rigid connections for between beams and columns.

The connection behavior is represented by its moment-rotation relationship.Extensive experimental works on connections have been performed, and a large body of moment and rotation database has been collected (Goverdhan 1983, Nethercot 1985a, Kishi and Chen 1986, and Chen and Kishi 1989). These experiments have been reported in [1] and are included in the steel connection databases [4, 5]. They are used next in the feed-forward BP-ANN model.

The experimental work for semi-rigid connections of between beams and columns has yet been extensive, and curve-fitting techniques have therefore been developed as alternatives to analytical models.Such methods are attractive,because generally they provide simpler ways of predicting behaviour than analytical models.Also, they may be more reliable within certain ranges, because of simplifying assumptions in an analytical model.A further alternative though is to use experimental data for semi-rigid connections to train an BP-ANN to predict semi-rigid connections behaviour.

Extensive experimental tests have been conducted on the commonly used connections, and a large collection of test data reported (Kishi and Chen 1986).The experimental results of semi-rigid steel connections have been taken from the databases described in [3, 5]. In particular, the case of the top and seat angles with double web angles, beam-to-column connections with the angle bolted to both the beam and the column has been considered here.The experimental database considered here concern the case of top and seat angles with double web angles beam-to-column bolted connections.

On one hand, the stiffness of a connection is dependent on the geometric parameters, such as plate or angle thickness, beam and column dimensions, the number of bolts in connection, etc., on the other hand, The moment-rotation relationship is nonlinear for all types of semirigid connections over the entire range of loading, and varies depending on connection semirigidity, i.e., the more rigid the connection, the smaller the relative rotation.

A BP-ANN such as the one described previously is able to learn an input and output relation between appropriately preprocessed data. All input and output variables should include in the range of the activation. MATLAB programs were used and developed to construct the BP-ANN prediction model. BP-ANN prediction training data of beam-to-column connections for the top and seat angles with double web angles were obtained from the experiments database already summarized [3, 5]. The ten variables that were used as the input vectors correspond to the column, beam and the connection. They are: depth of column section; flange thickness of column section; web thickness of column section; flange breadth of beam section; depth of beam section; the number of bolts in connection; thickness of angles; the ultimate bending moments at beam ends; relative rotations at beam ends; initial stiffness for semi-rigid connections . The outputs are the joint ultimate bending moments at beam ends and the corresponding joint relative rotation between beams and columns. The experimentally measured moment-rotation relationship of semirigid connections between beams and columns for steel structures are first preprocessed in a form suitable for neural network treatment. These database are next used for training a multilayer BP-ANN. The trained back-propagation artificial neural network provides the better prediction model, with satisfactory accuracy as shown in Fig. 3.

Intellectualized prediction models using BP-ANN, can provide an alternative to conventional methods of determining the moment and rotation curves for beam-to-column rigidity connections by providing an inside relationship in the form of generalizations 


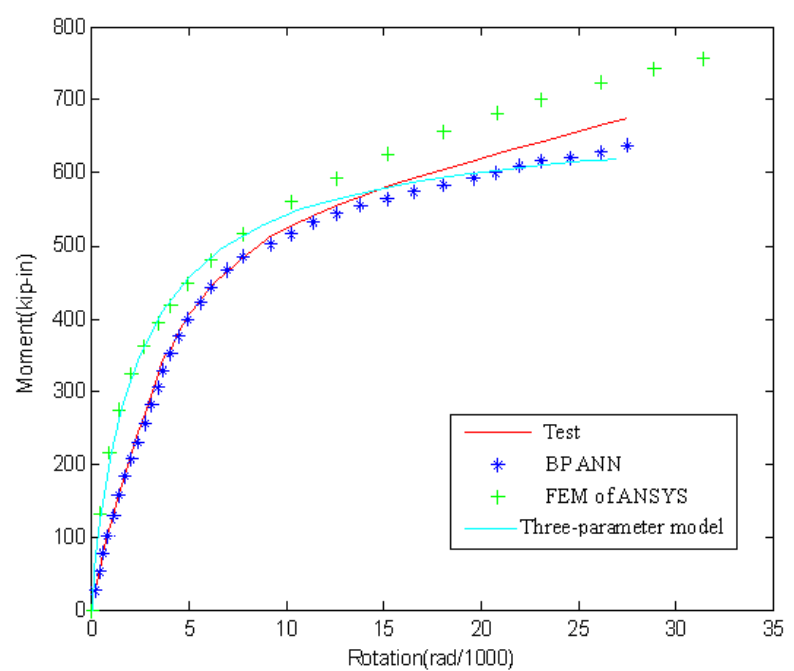

Fig. 3 The moment-rotation curves predicted by BP-ANN.

between the parameters involved.Thereby obtaining a more accuracy moment and rotation curves for rigidity connections by extracting information directly from the experimental results.

\section{Conclusions}

Steel frames for buildings are usually designed on the basis that beam-to-column connections are either pinned or rigid. The actual response, however, will usually fall somewhere between these two extremes, giving what is generally termed "semi-rigid" behaviour. This is represented by a moment-rotation curve, relating the moment transmitted by the connection to the relative rotation between the beam end and the adjacent column.

BP-ANN are information processing systems composed of simple processing elements with nonlinear behavior based on previous investigations, the intellectualized analytical model of the moment rotation relationship is proposed in the semirigid connection between beam and column using the feed-forward BP-ANN technique, in this paper. The intellectualized analytical model from experimental results based on BP-ANN is more reliable and it is the better choice of the moment-rotation curves for beam-to-column semirigid connection. The neural network model treats all the parameters describing the experimental curves with the same accuracy. The trained back-propagation artificial neural network provides the better prediction model, with satisfactory accuracy.

\section{Acknowledgements}

The work presented in this paper is supported by funds of Bureau of Guangzhou Education (Grant No. 08C056), and Science and Technology Project for Ministry of Construction, the People's Republic of China (Grant No. 2008-k2-36, 2010-K3-33). The authors wish to appreciate these financial supports.

\section{References}

[1] W. F. Chen and E. M. Lui, Stability Design of Steel Frames, Boca Raton, CRC Press, USA, 1991, pp. 165-182.

[2] S. Haykin, Neural Networks: A Comprehensive Foundation, New York: Prentice Hall, 1998.

[3] W. F. Chen and S. Toma, Advanced Analysis of Steel Frame: Theory, Software, Application, CRC Press Inc. USA, 1994.

[4] K. M. Abdalla and W. F. Chen, Expanded database of semi-rigid steel connections, Compurers \& Structures 56 (1995) (4) 553-564.

[5] N. Kishi and W. F. Chen, Database of steel beam-to-colunm connections, Vols I and II. Structural Engineering Report No. CE-STR-86-20, School of Civil Engineering, Purdue University, West Lafayette, IN, 1986.

[6] J. Kim, J. Ghaboussi and A. S. Elnashai, Mechanical and informational modeling of steel beam-to-column connections, Engineering Structures (32) 2010 (2) 449-458. 